\title{
Editorial: \\ Taking Stock: Elder Abuse Research in Canada
}

This special issue of the Canadian Journal on Aging is devoted to the current research in the area of elder abuse and neglect in Canada. While it would be exciting to announce that this is a first in Canadian history, it would not be an accurate statement. Enterprising Canadian researchers and practitioners, with the help of accommodating Americans, managed to appropriate a whole issue of the Journal of Elder Abuse and Neglect in 1992 to showcase the most recent research in Canada on elder abuse and neglect. That issue included the landmark national survey carried out by Elizabeth Podnieks which revealed that 4 per cent of elderly Canadians living in private dwellings have experienced some form of abuse and neglect -2.5 per cent reporting material abuse, 1.4 per cent experiencing chronic verbal aggression, .5 per cent reporting physical violence and .4 per cent neglect (Podnieks, 1992). With that special edition of the American journal, the first era of Canadian research on elder abuse and neglect came to a propitious end. Researchers, spurred on by Canadian practitioners, had successfully brought to the attention of the Canadian public an unacceptable social problem.

The first prevalence studies by Bélanger (1981) and Grandmaison (1988) in Montreal, Shell (1982) and King (1984) in Manitoba, Haley (1984) in Nova Scotia, Stevenson (1985) in Alberta and by the Ministry of Community and Social Services (1985) in Ontario as precursors to the Podnieks survey, established that all was not well amongst a certain proportion of Canada's seniors. The first Canadian book on abuse of the elderly by Schlesinger and Schlesinger (1988) helped to alert the unsuspecting to the issue and a number of legal responses to the problem were implemented as early as 1973 in Newfoundland. A spirited discussion of adult protection legislation and the pros and cons of mandatory reporting of abuse dominated the academic discourse in the 1980s and influenced the development of reforms in the adult protection field. In the 1980 s the federal government became involved in funding various research, educational, and intervention initiatives and as a result, at least six major research projects were under way by the end of the 1980 s and the beginning of the 1990s.

These developments of the 1980s have come to fruition in the 1990s and have set the stage for a new era in the investigation of elder abuse in Canada. Unlike the previous period which was devoted to providing irrefutable evidence of the existence of elder abuse - sometimes through an overstatement of the problem - the current era is rooted in solid research that even now informs practice interventions, the formulation of policy and the evolution of Canadian legislation. Some have argued that Canada has sadly lagged be-

Canadian Journal on Aging / La Revue canadienne du vieillissement / Vol. 14 sup.2 1995, 1-13 
hind the United States in its treatment of elder abuse and neglect but we would argue that Canada has been very wise in its approach to the problem. There has not been a wholesale adoption of the highly criticized adult protection legislation found in the United States but rather a thoughtful testing of different approaches in different provinces. Practitioners have worked together in multidisciplinary teams with a certain mutual respect and sensitivity appropriate to the absence of practice knowledge while governments have been swift in their support of research and educational endeavours, thereby encouraging a whole new generation of researchers. This special edition provides a sampling of this work which comprises the foundation for future research ventures and points to some of the serious issues that all Canadians will have to grapple with as we move into a definitely leaner, but, hopefully not meaner 21st century.

In the first article, Pittaway, Westhues and Peressini, using secondary data, examine the links between the usual theories used to explain elder abuse and neglect and the risk factors believed to place older persons or their caregivers in danger of abuse and neglect. When the discussion turns to an explanation of elder abuse, one of the deficiencies in the elder abuse research becomes immediately apparent. Incidence research (number of new occurrences of abuse within a specific time) is virtually non-existent in Canada and the United States, yet it is the most effective mechanism for examining the causation of elder abuse and neglect and for testing the effectiveness of prevention programs. Incidence figures also tells us whether the problem is getting better or worse, currently a matter of conjecture in Canada.

Recognizing the limitations in their data, Pittaway and her colleagues find that different factors correlate with different types of abuse. They suggest that a comprehensive, all-inclusive explanation of the phenomena is probably not possible, a conclusion supported in the American literature. Because elder abuse is so complex, a number of different theories are required to explain the different dimensions. The need for a variety of theories has not, however, generated much interest. A scan of the elder abuse literature suggests a surprising absence of new theories. Although feminist theory, in its many forms, is a highly desirable addition to the field, it is not really a new theory, but rather a recently borrowed perspective from the field of family violence. Strangely enough, the most prevalent form of abuse in Canada - financial abuse - receives even less academic attention in terms of theorizing than the other forms of abuse. Today, mounting research evidence emphasizes the interactive aspects of elder abuse, and supports distinctions between senior-directed, senior-generated and mutual abuse but there have been few attempts at theory building in light of this recent information. Theory building, sometimes viewed with skepticism, is ignored at our own peril, since theory determines what actions should be taken to ameliorate abuse and neglect and is reflected in social policy and legislation.

Although advances on the theoretical front have been slow, there is considerable promise in the development of screening protocols and the Reis and Nahmiash article in this issue is an excellent example of this activity. 
In their study, they test the validity of the Caregiver Abuse Screen (CASE), a short, eight item, written measure, completed by caregivers in the community. Their carefully crafted study provides support for the ability of the screen to distinguish between the abused and the non-abused and will be a most welcome tool in the armamentarium of practitioners in the battle against elder abuse. Having said this, there is still considerable psychometric work to be done in Canada in developing screens for identifying abuse and for identifying the type of person at risk for abuse, not to mention the need for a valid classification protocol to assist in proper diagnosis and intervention.

In a similar vein, the paper by Stones and Pittman provides a further example of the research activity devoted to developing the psychometric properties of a measure of attitudes toward elder abuse. Using two studies, Stones and Pittman confirm that the Elder Abuse Attitude Test (EAAT) is a psychometrically sound measure of attitudinal differences towards elder abuse. At the same time, the authors touch on some fascinating findings that undoubtedly will be controversial in some quarters. They find that people more prone to extreme attitudes have stronger convictions about the responsibility of society to end abuse; they observe that attitude extremity is related to simpler and conflicting belief structures and that such trends predict more extreme attitudes to those outside one's own group. For example, younger practitioners rated examples of elder abuse more severely than seniors. These findings, for example, might help to explain why some abused seniors choose to live at greater risk than others would choose for them. The researchers wonder if the stronger advocates against abuse are also those with simpler and conflicting belief structures. Upon application, their findings may help to explain the criticism directed at the earlier abuse research which was perceived as being an exaggeration and overstatement of the problem and demanding draconian action.

The paper by Aronson, Thornewell and Williams, is a case in point about the significance of making an accurate classification of the type of abuse. These authors provide a first person account of a woman who left her abusive husband in her seventies. The paper argues for the unveiling of elder abuse for what it is in many situations - wife assault, a hidden problem because of gender neutrality in gerontology and age blindness in women's studies. Even though the existing prevalence studies clearly indicate that spouse abuse is an important component of elder abuse, there is still a certain reluctance on the part of gerontologists to move beyond the highly popular situational model which views mistreatment as an irrational response to a stressful caregiving situation. The situational model has its roots in the family violence literature, specifically relating to child welfare, and could be considered a competing model to feminist interpretations of violence which are undeniably better equipped to identify wife abuse. Operating from the situational model, gerontologists may be reluctant to identify spouses as abusers because family caregivers, namely women, have proven to be the mainstay of community care for the elderly; Canadian research shows, 
without a doubt, that family members are willing and responsible caregivers making it unattractive to accuse them of abuse; and spouse abuse sometimes requires different solutions that are often not within the intervention lexicon of gerontologists (McDonald, Hornick, Robertson, \& Wallace, 1991).

Whatever the reasons for the hesitancy of gerontologists to recognize wife abuse "grown old" or, for that matter, "new abuse" amongst older wives, the application of feminist theories to all forms of spouse abuse is still a debated issue. For example, the small but growing body of research on gay and lesbian domestic violence has raised questions about gender-based theories of partner violence (Renzetti, 1994). The real culprit, it is argued, is the power imbalance between the partners. Feminist theories then, might be extended to explain both female and male spouse abuse if developed in these terms because presently, they provide only a partial account of elder abuse, since older men are just as likely as older women to be abused. The refined theories may also have direct application to other older vulnerable groups, such as those with different ethnic membership and the very frail elderly, possibilities raised by Aronson et al. Most importantly, the feminist theories direct attention to the wider implications of gender, race, ethnicity, and class, structural factors all too often overlooked in the study of elder abuse.

The last three papers in this special edition address the ongoing concerns that Canadians have about adult protection legislation. The Gordon article provides a description of the evolution of Canadian legislation relevant to elder abuse and neglect by identifying what he considers to be three successive waves in guardianship law reform. The third wave of reform, the recent enactment of new omnibus adult guardianship statutes that include adult protection provisions, is what concerns this author. He draws attention to three potential problems that most Canadians would be foolish to overlook, especially in light of the articles by Poirier and by Bond, Penner and Yellen which lend empirical support to Gordon's observations. The first concern relates to bringing together court-ordered guardianship and protective provisions in the same statutes. The combination may have positive aspects but, on the negative side, court-ordered guardianship, an intrusive solution to elder abuse, is in danger of becoming routine. One of the suggestions made by Gordon to prevent this problem is to preserve the role of the courts. The Poirier paper, which compares the outcomes for clients subject to the adult protection legislation in New Brunswick, finds that the most important factor in determining whether or not protective measures are ordered is whether or not the client has a lawyer. The data, based on the analyses of court files from 1983 to 1989 , clearly show that aged clients fare better with legal representation. At the same time, Poirier also finds that the legal philosophy of the judge has a significant effect on the outcome of the case. The danger here is that some judges more readily accept the questionable social intervention philosophy characteristic of adult protection legislation.

The second weak point noted by Gordon in the newer legislation pertains to the prominent role advocacy plays in protecting the abused adult. In times of fiscal restraint there is a genuine possibility that these sophisticated serv- 
ices, which are targeted at a special population, will suffer at the hands of politicians as they slash budgets in the name of the deficit. A central finding of the Bond, Penner and Yellen paper, which surveyed professionals about the legislation regarding elder abuse, was the major concern respondents expressed about the lack of funds to administer the legislation and to provide services to abused older persons - and this was before governments became serious about their intent to reduce the debt load! The last concern of Gordon, the possibility of an increase in elder abuse and neglect as a result of the introduction of legislation that removes certain safeguards to achieve the loftier goal of empowerment, has no empirical referent. The lack of research on the trade-offs between empowerment (borrowed from the mental disability movement) and protection of the older person is reminiscent of the hasty implementation of mandatory reporting provisions, (borrowed from child welfare) before anyone really knew what the influence of this legislation would be. Empowerment of the elderly has become an influential approach in gerontology but we have to be very careful that it does not become a euphemism for no service and/or no protection.

In taking stock of the Canadian research on elder abuse and neglect, then, it is heartening to see the substantial progress that has been made in a relatively short period of time by a small cadre of scholars. This is not to say, however, that abuse and neglect is receiving the research attention it deserves or that the degree of seriousness it warrants is recognized. Canadian researchers have a daunting program of research before them which will have to be undertaken during a period of ever-diminishing research dollars. Unfortunately, the research waiting to be done will be expensive - at minimum, we require an incidence study of elder abuse; a prevalence study of elder abuse in institutions to see who is abusing whom; a prevalence study of abuse in the delivery of home-care services; case control studies to determine risk factors for abuse; more evaluative research of both the qualitative and quantitative type of what works in practice; the ongoing development and testing of screening measures and classification inventories; and direct evaluations of the latest round of legislative changes. The research will have to be done in a climate wherein the use and relevance of research is often regarded with misgiving, the underlying belief being that the money would be better spent on prevention and service provision. What is more, elder abuse is one of many equally worthy social problems and will be part of a broader competitive process as specialized funding agencies are dismantled.

Are the obstacles insurmountable? We think not. Research will be necessary to help ration the use of scarce resources on interventions, services and social policies through demonstrating their effectiveness. Most Canadians care about the fate of their older citizens; governments, even though financially constrained, are likely to continue to be supportive of practical research; abuse will no doubt take its collaborative place in the life cycle approach to the study of violence and Canadian researchers will continue to demonstrate their creativity and resourcefulness. 


\section{References}

Bélanger, L. (1981). The types of violence the elderly are victims of: Results of a survey done with personnel working with the elderly. Paper presented at the 34th Annual Scientific Meeting of the Gerontological Society of America, Toronto, Canada.

Grandmaison, A. (1988). Protection des personnes âgées: Étude exploratoire de la violence á l'égard de la clientèle des personnes âgées. Unpublished manuscript. Montréal, Centre de Services Sociaux du Montréal Métropolitain (CSSMM).

Haley, R.C. (1984). Elder abuse/neglect. Halifax: Department of Social Services.

King, N.R. (1984). Exploitation and abuse of older family members: An overview of the problem. In J.J. Costa (Ed.), Abuse of the elderly: A guide to resources and services (pp. 56-68). Lexington, MA: Lexington Books.

McDonald, L., Hornick, J., Robertson, G., \& Wallace, J. (1991). Elder abuse and neglect in Canada. Toronto: Butterworths.

Ministry of Community and Social Services. (1985). Report of a survey of elder abuse in the community. Toronto: Standing Committee on Social Development, Government of Ontario.

Podnieks, E. (1992). National survey on Abuse of the elderly in Canada. Journal of Elder Abuse and Neglect, 4, 5-58.

Renzetti, C.M. (1994). On dancing with a bear: Reflections on some of the current debates among domestic violence theorists. Violence and Victims, 9, 195-200.

Schlesinger, B., \& Schlesinger, R. (1988) Abuse of the Elderly: Issues and Annotated Bibliography. Toronto: University of Toronto Press.

Shell, D.J. (1982). Protection of the elderly: A study of elder abuse. Report of the Manitoba Council on Aging. Winnipeg: Association of Gerontology.

Stevenson, C. (1985). Family Abuse of the Elderly in Alberta. Report of the Government of Alberta Social Services and Community Health, Senior Citizen's Bureau. 